\title{
Shape optimisation of the sharp-heeled Kaplan draft tube: Part I - performance evaluation using Computational Fluid Dynamics
}

\author{
S. J. Daniels ${ }^{\mathrm{a}}$, A. A. M. Rahat ${ }^{\mathrm{b}}$, G. R. Tabor ${ }^{\mathrm{a}}$, J. E. Fieldsend ${ }^{\mathrm{a}}$, R. M. \\ Everson $^{\mathrm{a}}$ \\ ${ }^{a}$ College of Engineering, Mathematics and Physical Sciences, University of Exeter \\ ${ }^{b}$ School of Computing, Electronics and Mathematics, University of Pymouth
}

\begin{abstract}
\end{abstract}
\section{Keywords: Hölleforsen-Kaplan draft tube, Pressure recovery, Grid} Convergence Index, cfMesh, Catmull-Rom Splines.

Email address: S.Daniels@exeter.ac.uk (S. J. Daniels) 


\section{Introduction}

The performance of a hydraulic turbine is significantly affected by the efficiency of its draft tube, which serves the following functions:

- to recover energy, by converting some of the kinetic energy leaving the runner into static head that would otherwise be lost in the absence of a draft tube;

- to position the turbine runner above or below the tail water level to avoid cavitation, without affecting the net-head.

Several factors make the design of the draft tube a daunting task. The flow itself, largely decelerating, is subject to viscous turbulent effects (such as flow separation) which reduce its effectiveness. To make matters worse, some designs are often made more complicated by the inclusion of an approximately $90^{\circ}$ bend (elbow-type) to improve powerhouse compactness and to minimise construction costs. Furthermore, the outflow cross-section is often rectangular, while the inflow cross-section is circular to couple with the runner. Thus, the geometry of the draft tube design needs to be thought out very carefully to achieve the best possible compromise between hydraulic efficiency and construction costs. This leads to a large number of design parameters which could potentially be changed to alter and optimise its efficiency.

Fundamentally, factors which alter the draft tube's performance are its geometrical shape, and the velocity distribution (profiles) at the inflow. So far, the design of the draft tube has been tempered through experimental observations and semi-empirical formulae of established geometries (notably: [1]). To explore potential new designs, Computational Fluid Dynamics (CFD) has proved to be a powerful tool for the engineer, allowing for comprehensive analysis of complex flowfields where experimental work provides limited insight. CFD becomes especially appealing when combined with a global optimisation method which may significantly reduce the number of evaluations during the design cycle. Consequently, there is a need for developing an accurate and robust CFD approach, together with an efficient optimisation strategy.

Parameter-based shape optimisation is based on the philosophy that, any geometry in all its complexity and details, can be described by a group of parameters (control points), allowing the geometry to be suitably modified to improve its performance. Through this approach, it is easy to co-relate the 
impact of a parameter's value on the design objectives. More importantly, this approach allows the exploration of large global design spaces without any conceptual barriers. However, cases involving such unconstrained design spaces may result in complex geometries, potentially compromising the accuracy of the objective functions depending on the fidelity of the CFD methodology.

CFD requires the solution of a set of Partial Differential Equations (PDEs) describing the physics of fluid flow. This is typically achieved using a discretisation method, in which a grid is constructed across the fluid continuum, and the PDEs are solved algebraically within each cell. Cell quality issues can impede the accuracy of the eventual solution, even to the point where the solver diverges and no solution is generated; they can also significantly affect the level of computational work (i.e. number of iterations) necessary to reach the solution. Thus, grid generation is commonly recognised as one of the main challenges in CFD, which in itself has motivated the use of optimisation techniques to improve the overall grid quality (e.g. [2]). Moreover, for automated shape optimisation, large perturbations of the geometry's surface will require the Computer-Aided Design (CAD) model and CFD grid to be reconstructed for each evaluation (e.g. [3, 4, 5]), rather than redistribute the existing grid within the domain. However, despite their potential, reports on the application and efficacy of automated CAD and grid regeneration techniques for shape optimisation are largely absent in the literature.

In the context of draft tube shape optimisation, reports have often employed the use of commercial software to reconstruct the CAD and grid for each evaluation. Marjavaara and Lundström [6] and Hellström et al. [7] investigated the heel curvature effects on the draft tube efficiency using the commercial software I-deas NX 10 and ICEM CFD Hexa to construct the CAD geometry and CFD grid respectively. While grid sensitivity analysis was carried out, neither the topology of their base grid or method of refinement were reported. Galván et al. [8] employed ANSYS Fluent to construct a block-structured grid while uniformly refining all vertices for their sensitivity study. The above papers employ Richardson extrapolation of the grid-solution convergence to estimate the uncertainty [9, 10]. However, they report oscillating convergence issues (possibly indicating a topological problem within the grid [11, 12]) - the nature of these issues remains uncertain. With an increasing interest in automatically optimising the shape of the draft tube with more unconventional design features (see [13]), the sensitivity of the CFD grid resolution for these draft tube designs should be investigated. 
Thus, in the present work, the use of an open-source grid regenerator and consistent CFD methodology is used to assess the efficiency of number of proposed draft tube designs from the literature, and to gain a deeper insight into the uncertainty of the results to the grid resolution. Overall, this analysis will aid future CFD applications to draft tube designs in association with automated shape opimisation.

\subsection{Base draft tube geometry}

Elbow-type draft tubes are widely used in conjunction with vertical Kaplan and Francis turbines, due to their lower excavation cost and greater potential for pressure recovery. The two most common draft tube designs reported in the literature are the sharp-heeled (e.g., [14, 15]), and underground (e.g. [16]) types. The former encompasses a large group of draft tubes that were installed in Swedish hydropower plants during the 1950s. The base geometry considered in the present work is a 1:11 scaled model of the Hölleforsen-Kaplan draft tube, constructed in 1949. This design has served extensively as a benchmark test case for both experimental and numerical studies in the literature - largely through the European Research Community On Flow, Turbulence And Combustion (ERCOFTAC) Turbine99 Workshop series [17, 14, 18]. A schematic of the draft tube geometry is shown in Fig.1.

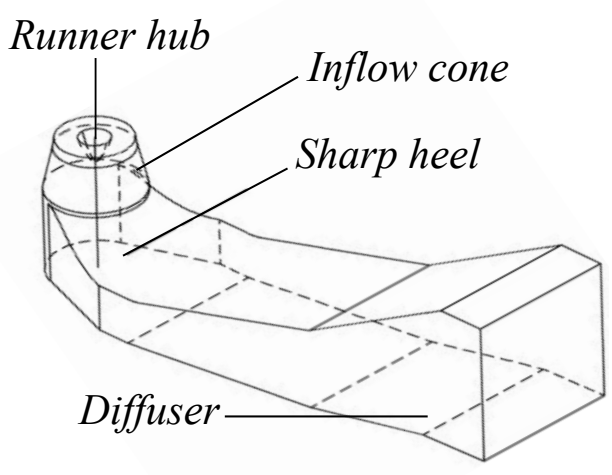

Figure 1: Schematic of the sharp-heeled Hölleforsen-Kaplan draft tube.

\subsection{Paper Overview}

With the overarching aim to improve the draft tube performance over two consecutive papers, this first contribution will address the following topics: 
- to examine the draft tube efficiency based on the method of estimation;

- to investigate the performance of proposed designs for the elbow-type draft tube;

- to assess the uncertainty of performance measures relating to the grid resolution for various draft tube designs.

The structure of this paper reflects the stages of work undertaken towards achieving the above goals. \$2 outlines the overall methodology used for assessing the flow through the draft tube, starting with the simulation setup in 82.1 . The methods of measuring the performance of the draft tube is outlined in 32.2 . This is followed by the methodology for the automatic grid regeneration in $\$ 2.3$. The proposed CFD methodology is subsequently validated using the sharp-heeled Hölleforsen-Kaplan draft tube in $\$ 2.4$ with a discussion concerning the 1st topic and overall fidelity of the CFD approach. This is examined further in $\$ 2.5$ in which the Grid Convergence Index (GCI) method [12] is used to estimate the uncertainty associated to the grid resolution. \$3 applies the above CFD methodology to a number of proposed draft tube designs from the literature. A study of the inflow cone and outer-heel design on the draft tube performance is carried out in $\$ 3.1$ and $\$ 3.2$ respectively, addressing the 2nd topic of this paper. GCI analysis is also applied to these designs following the 3rd topic. Finally, in $\$ 4$, the observations, and premise for future work are summarised.

\section{Numerical methodology}

\subsection{CFD setup}

The CFD simulations in this work were performed using the open-source $\mathrm{C}++$ code OpenFOAM-4.x. Since its public release in 2004, OpenFOAM has been the subject of many validation publications, including the flow through the draft tube considered in this work (e.g. [19, 20]). The fluid flow was modelled using the Reynolds-Averaged Navier-Stokes (RANS) equations. These equations can be derived by substituting mean and fluctuating components of the flowfield variables into the incompressible Navier-Stokes equations:

The continuity equation:

$$
\frac{\partial U_{i}}{\partial x_{i}}=0 .
$$


154

The momentum equations:

$$
\frac{\partial U_{i}}{\partial t}+U_{j} \frac{\partial U_{i}}{\partial x_{j}}=-\frac{\partial \bar{p} / \rho}{\partial x_{i}}+\nu \frac{\partial^{2} U_{i}}{\partial x_{j} \partial x_{j}}-\frac{\partial}{\partial x_{j}}\left(\overline{u_{i}^{\prime} u_{j}^{\prime}}\right),
$$

where

$$
\overline{u_{i}^{\prime} u_{j}^{\prime}}=\nu_{t}\left(\frac{\partial U_{i}}{\partial x_{j}}+\frac{\partial U_{j}}{\partial x_{i}}\right)-\frac{1}{3} \overline{u_{i}^{\prime} u_{i}^{\prime}} \delta_{i j} .
$$

$U$ and $\bar{p}$ are the averaged velocity and static pressure respectively, and $u^{\prime}$ is the fluctuating component of velocity. $\rho$ and $\nu$ are the density and kinematic viscosity of the fluid. The standard $k-\epsilon$ model was used for the calculation of the turbulent viscosity by the relation $\nu_{t}=C_{\mu} k^{2} / \epsilon$, where $k$ is the turbulent kinetic energy, and $\epsilon$ is the rate of dissipation. The $k$ and $\epsilon$ transport equations are described:

$$
\frac{\partial k}{\partial t}+\frac{\partial}{\partial x_{i}}\left(U_{i} k\right)=\frac{\partial}{\partial x_{j}}\left[\left(\nu+\frac{\nu_{t}}{\sigma_{k}}\right) \frac{\partial k}{\partial x_{j}}\right]-\overline{u_{i}^{\prime} u_{j}^{\prime}} \frac{\partial U_{j}}{\partial x_{i}}-\epsilon,
$$

$$
\frac{\partial \epsilon}{\partial t}+\frac{\partial}{\partial x_{i}}\left(U_{i} \epsilon\right)=\frac{\partial}{\partial x_{j}}\left[\left(\nu+\frac{\nu_{t}}{\sigma_{\epsilon}}\right) \frac{\partial \epsilon}{\partial x_{j}}\right]-\frac{\epsilon}{k}\left(C_{1 \epsilon} \overline{u_{i}^{\prime} u_{j}^{\prime}} \frac{\partial U_{j}}{\partial x_{i}}+C_{2 \epsilon} \epsilon\right),
$$

where the associated empirical coefficients are defined in Table 1.

$$
\begin{array}{|ccccc|}
\hline C_{1 \epsilon} & C_{2 \epsilon} & C_{\mu} & \sigma_{k} & \sigma_{\epsilon} \\
\hline \hline 1.44 & 1.92 & 0.09 & 1 & 1.3 \\
\hline
\end{array}
$$

Table 1: Empirical constants for used for the standard $k-\epsilon$ turbulence model.

The suitability of the $k-\epsilon$ turbulence model in simulating the swirling flow and near-wall modelling along the draft tube has been extensively studied in the Turbine-99 workshop series and independent publications (e.g. [18, 21, 22, 13]). The Finite Volume Method was used to integrate the above equations [23]. The second-order central difference scheme was used to discretise the diffusion terms, and the second-order upwind difference was adopted for the convection term. For the unsteady simulations, a first-order implicit scheme (Euler) for the temporal discretisation was employed; in such cases, the PISO algorithm [24] was adopted for the velocity-pressure coupling, with 
the number of pressure correctors set to 2. For the steady-state calculations, the SIMPLE algorithm [25] was used, with under-relaxation factors $0.7,0.3$, and 0.7 for the velocity, pressure, and turbulence quantities respectively. The generalised Geometric-Algebraic Multi-Grid solver was used to solve the pressure field, while the Gauss-Seidel linear solver was used for the remaining field variables.

The boundary conditions in the present work are chosen to reproduce those specified by the organisers of the 2nd Turbine-99 Workshop [14]. At the outflow, all field variables, excluding pressure, are specified as a zero-normal gradient, i.e., it is assumed that the field is fully developed at the outlet. Moreover, an extension to the outflow of $2 m$ was applied to the geometry to avoid any backflow at the outflow plane, and to ensure convergence of the solution. For the draft tube walls, a no-slip condition is applied for the velocity, and a zero-normal gradient condition for pressure; a rotational velocity was applied to the runner-hub in accordance to the turbine rotation. At the inflow, a swirl flow was imposed to represent the discharge from the Kaplan turbine. The axial $(U)$ and tangential $(W)$ velocity components from LaserDoppler-Anemometry (LDA) measurements [14, 26] are linearly interpolated onto the CFD boundary. Data for the radial velocity, Reynolds stresses, and turbulent length scales were not reported and had to be approximated. The radial velocity $(V)$ distribution at the inflow was assumed to be attached to the runner-hub and the draft tube walls, as described through the function proposed by Cervantes et al. [18]:

$$
V(r)=U(r) \tan (\theta),
$$

where

$$
\theta=\theta_{\text {cone }}+\left(\frac{\theta_{\text {wall }}-\theta_{\text {cone }}}{R_{\text {wall }}-R_{\text {cone }}}\right)\left(r-R_{\text {cone }}\right),
$$

with $R_{\text {cone }} \leq r \leq R_{\text {wall }}, \theta_{\text {cone }}=-12.8^{\circ}$ and $\theta_{\text {wall }}=2.8^{\circ}$ for the geometry considered [18. The unknown turbulent quantities at the inflow are assumed: $\overline{v^{\prime}}=\overline{w^{\prime}}$, and $\overline{u^{\prime} u^{\prime}}=\overline{u^{\prime} v^{\prime}}=\overline{u^{\prime} w^{\prime}}$ in accordance to the modelling specifications provided in the 2 nd Turbine-99 workshop [14]. The quantities for $k$ and $\epsilon$ at the inflow boundary were estimated by the following expressions:

$$
k=\frac{1}{2}\left(\overline{u_{i}^{\prime} u_{i}^{\prime}}\right)=\frac{3}{2}\left(\left(\frac{Q}{A_{\text {in }}}\right) I\right)^{2},
$$

$$
\epsilon=\frac{C_{\mu}^{\frac{3}{4}} k^{\frac{3}{2}}}{l_{\epsilon}} ; \quad l_{\epsilon}=0.1\left(R_{\text {wall }}-R_{\text {cone }}\right),
$$


where $Q$ and $A_{i n}$ are the volumetric discharge and cross-sectional area of the inflow, and $I=u^{\prime} /\left(Q / A_{i n}\right)$ is the turbulence intensity - estimated as $10 \%$ from the experimental data by Andersson and Cervantes [26]. $l_{\epsilon}$ is a constant length scale, recommended to be between 1-10\% of the hydraulic diameter [27, 14]. The operating conditions for the Kaplan turbine were set at the ' $T(n)$ ' mode [18] detailed in Table 2.

\begin{tabular}{|cccc|}
\hline Operating Condition & $N(\mathrm{rpm})$ & $Q\left(\mathrm{~m}^{3} / \mathrm{s}\right)$ & $\operatorname{Re}_{D}\left(10^{6}\right)$ \\
\hline \hline$T(n)$ & 595 & 0.522 & 1.329 \\
\hline
\end{tabular}

Table 2: Kaplan turbine operating mode ' $T(n)$ '. $N$ is the rotational speed of the turbine, and Reynolds number $\operatorname{Re}_{D}=\left(Q / A_{i n}\right) D_{0} / \nu\left(D_{0}=0.5 m[14)\right.$.
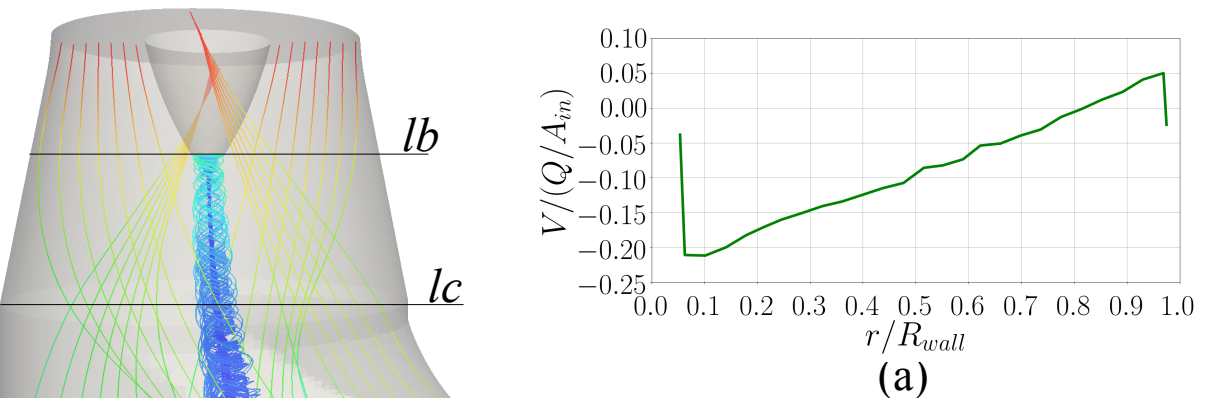

(a)

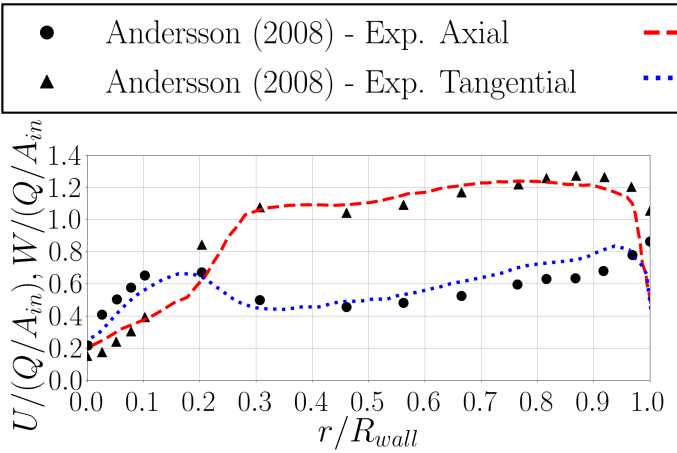

(b)

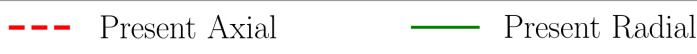
...... Present Tangential

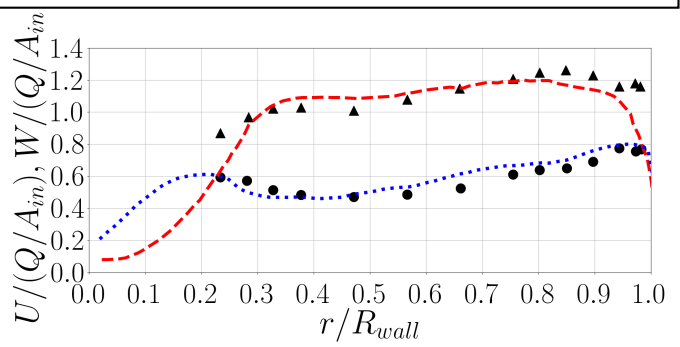

(c)

Figure 2: Comparison of the circumferentially averaged velocity components to experimental data from the literature at the two levels within the cone section; (a) Radial velocity at $l c$ level; (b) Axial and tangential components at $l b$ level; (c) Axial and tangential components at $l c$ level. The CFD profiles were derived from a steady-state simulation with grid resolution 'Mesh B' outlined in $\$ 2.3$.

Fig 2 shows the circumferentially-averaged velocity components at two 
levels of the inflow cone. The velocity components are normalised by the volumetric discharge at the inflow boundary. For comparison, the equivalent phase-averaged LDA measurements by Andersson and Cervantes [26] have also been plotted. It can be seen in this figure that the inflow methodology described above validates well with the equivalent experimental setup.

\subsection{Draft tube performance measures}

The main function of the draft tube is to recover kinetic energy from the turbine runner by increasing the pressure head. A typical measure of this conversion is given by the pressure recovery factor,

$$
C_{p}=\frac{1}{\frac{1}{2} \rho\left(\frac{Q}{A_{\text {in }}}\right)^{2}}\left[\frac{1}{A_{\text {out }}} \int_{A_{\text {out }}} \bar{p}_{\text {out }} d A_{\text {out }}-\frac{1}{A_{\text {in }}} \int_{A_{\text {in }}} \bar{p}_{\text {in }} d A_{\text {in }}\right],
$$

where $A$ denotes the cross-sectional area for the inflow $(i n)$ and outflow (out) boundaries respectively. Maximising $C_{p}$ is the primary objective in draft tube design. Conversely, another performance indicator, $\zeta$, expresses the energy that is converted to a form that can not be used during the operation of an energy producing, consuming, or conducting system (e.g. that due to frictional losses). Typically, $\zeta$ is defined [28]:

$$
\zeta_{1}=\frac{1}{\frac{1}{2} \rho\left(\frac{Q}{A_{\text {in }}}\right)^{2}}\left[\frac{1}{A_{\text {in }}} \int_{\text {in }} P_{t, \text { in }} d A_{\text {in }}-\frac{1}{A_{\text {out }}} \int_{\text {out }} P_{t, \text { out }} d A_{\text {out }}\right],
$$

where $P_{t}$ is the total pressure, i.e., $P_{t}=\bar{p}+0.5 \rho\left(U_{i}^{2}\right)$. Alternatively, the energy loss of the draft tube has been expressed in the literature in other forms [26]:

$$
\zeta_{2}=\frac{1}{\frac{1}{2} \rho\left(\frac{Q}{A_{\text {in }}}\right)^{2} U_{i} \cdot n}\left[\frac{1}{A_{\text {in }}} \int_{\text {in }} P_{t, \text { in }} U_{i} \cdot n d A_{\text {in }}+\frac{1}{A_{\text {out }}} \int_{\text {out }} P_{t, \text { out }} U_{i} \cdot n d A_{\text {out }}\right] \text {, }
$$

where $\cdot n$ indicates the component normal to the corresponding boundary - it should be noted that this component is negative at the inflow. The pressure recovery coefficient has also been reported in other forms [26]:

$$
C_{p}^{\prime}=\frac{1}{\frac{1}{2} \rho\left(\frac{Q}{A_{\text {in }}}\right)^{2} U_{i} \cdot n}\left[\frac{1}{A_{\text {out }}} \int_{A_{\text {out }}} \bar{p}_{\text {out }} U_{i} \cdot n d A_{\text {out }}-\frac{1}{A_{\text {in }}} \int_{A_{\text {in }}} \bar{p}_{\text {in }} U_{i} \cdot n d A_{\text {in }}\right],
$$


which, to the best of the authors' knowledge, has not yet been quantified in the literature. In this work, $C_{p}, \zeta_{1}$, and $\zeta_{2}$ will be used for validation of the proposed CFD methodology in $\$ 2.4$; $C_{p}^{\prime}$ on the other hand will be quantified to serve as benchmark data.

\subsection{Grid regeneration methodology}

The automated meshing utility cfMesh [29] was used to generate the CFD grid for each draft tube design. To construct the grid, cfMesh requires a closed manifold-surface - typically a stereolithography file. From this, a uniform hexahedral grid is generated within the enclosed surface. The internal grid is subsequently projected onto the manifold surface and a boundary layer grid is constructed towards the interior using a set of user-defined parameters. cfMesh also provides additional controls for the boundary layer quality, intended for situations where a large number of layers is required, or where the thickness is needed to vary smoothly - the majority of these parameters were kept as default. The chosen regions for local refinement were in the vicinity of the draft tube walls, inflow boundary, and the runner hub. Fig. 3 demonstrates 3 of the 9 key steps towards generating a predominately hexahedral grid $(\sim 95 \%)$, with occasional general polyhedral cells $(\sim 5 \%)$ in cumbersome regions of the domain.

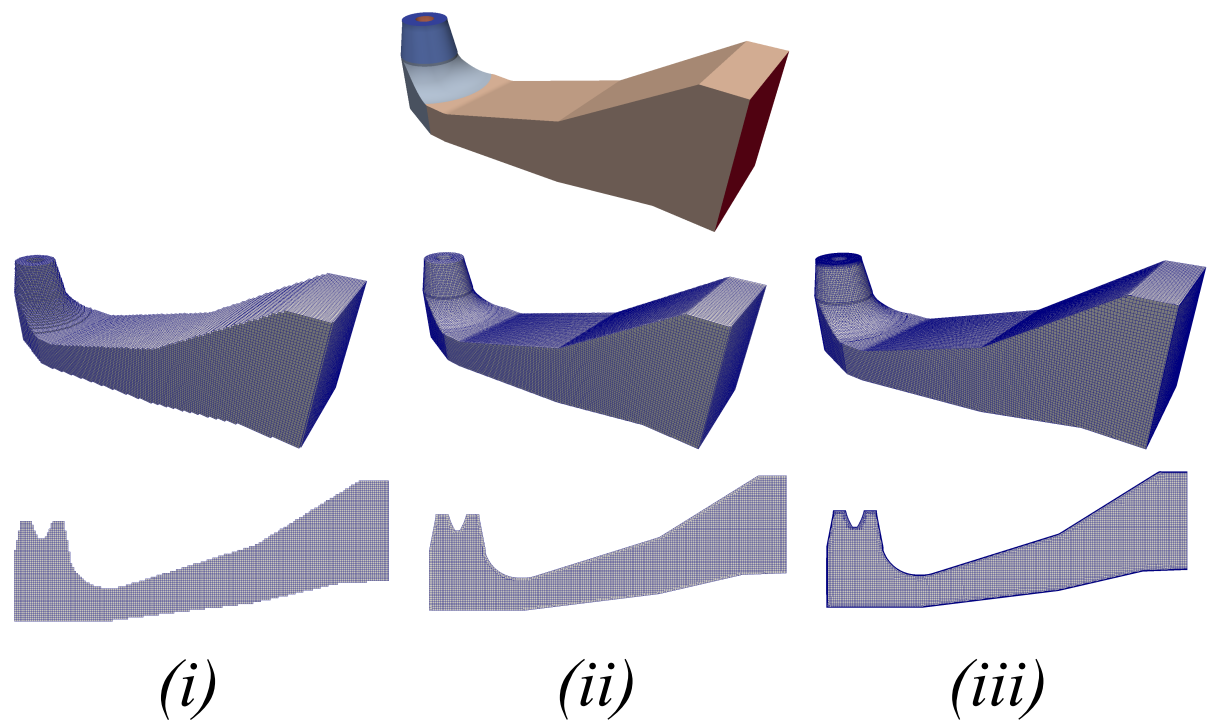

Figure 3: (Top) CAD geometry of the draft tube; (i) a uniform-hexahedral grid filling the internal domain; (ii) surface-projection of the internal grid onto the surrounding geometry; (iii) near-wall grid untanglement, boundary-layer construction and local region refinement. 
By experimentation, the most influential parameters needed for a grid independency study was reduced to a set of 3 :

- maxCellSize: defines the maximum cell size generated in the internal grid;

- localRefinement: prescribes the surface cell size on a specified boundary;

- maxFirstLayerThickness: prescribes the first wall-normal cell height to a specified boundary.

Four grids are generated varying the above parameters. The corresponding settings are shown in Table 3. 'Mesh A' has the coarsest resolution with the first cell height from the draft tube walls varying between $53 \leq y_{1}^{+} \leq 287$ (where $y_{1}^{+}=y_{1} u_{\tau} /\left(\nu+\nu_{t}\right), y_{1}$ is the cell-center height, and $u_{\tau}$ is the shear velocity). 'Mesh B' has a smaller maximum cell-size, refinement, and firstlayer boundary layer thickness than 'Mesh A' - the near-wall resolution was reduced to $33 \leq y_{1}^{+} \leq 187$. 'Mesh C' has the same maximum cell-size as 'Mesh B', and the same near-wall resolution as 'Mesh A'. Finally, 'Mesh D' increases the mesh resolution within the domain and has the same near-wall resolution as 'Mesh B'.

\begin{tabular}{|c|cc|c|c|}
\hline & \multicolumn{2}{|c|}{ Refinement } & Boundary-layer & \\
\hline Mesh & maxCellSize & localRefinement & maxFirstLayerThickness & Total no. cells \\
\hline \hline A & 0.02 & 0.025 & 0.035 & 1055311 \\
B & 0.015 & 0.0125 & 0.0175 & 2220036 \\
C & 0.015 & 0.0125 & 0.035 & 4280803 \\
D & 0.0075 & 0.005 & 0.0175 & 8491178 \\
\hline
\end{tabular}

Table 3: User-defined parameters used in cfMesh and resulting total number of cells for each CFD grid.

Steady-state simulations using the numerical setup described in $\$ 2.1$ were performed on the four grids. For comparison, a time-averaged transient simulation was performed on 'Mesh B'. The steady-state simulations were considered converged when the residuals for the flowfield variables descended below $10^{-6}$. For the unsteady simulation, the flowfield quantities were timeaveraged over a nondimensional time-period of $t^{*}=t\left(Q / A_{\text {in }}\right) / L=25(L$ is 
the length of the draft tube in the $x$-direction) with satisfactory convergence of the statistics. Fig 4 shows the profiles of the normalised wall pressure coefficient along the upper and lower walls along the centerline:

$$
C_{p w}=\frac{\bar{p}_{\text {wall }}-\bar{p}_{\text {in }, \text { wall }}}{\frac{1}{2} \rho\left(\frac{Q}{A_{\text {in }}}\right)^{2}},
$$

where $\bar{p}_{\text {wall }}$ is the local static pressure on the wall. For comparison, the experimental measurements by Andersson and Cervantes [26] and Čarija et al. 21] are also plotted along side the present results. It can be seen that the present results are consistent with the experimental data in the inflow cone region. Downstream, a large disparity can be seen around the heel section, especially along the lower wall, where attaining an accurate measurement for pressure is troublesome for both experimental and numerical approaches; for the former, this is demonstrated through the disparity of experimental measurements between Andersson and Carija et al., for the latter, the inability of CFD to validate in the corner region has been recorded for more advanced turbulence modelling approaches such as Detached-Eddy Simulation [30]. Finally, along the diffuser section, the present and experimental results return to a close agreement for both the upper and lower walls. Overall, although there is some deviation in the elbow section, the present results clearly agree the trend of the experimental measurements, and the CFD results show a consistent profile regardless of the cfMesh parameters pertaining to the near-wall resolution.

- Carija et al. (2006) - Exp. ---- Mesh A — Mesh C

- Andersson (2000) - Exp. ….. Mesh B -.-. Mesh D

-.-· Unsteady (Mesh B)
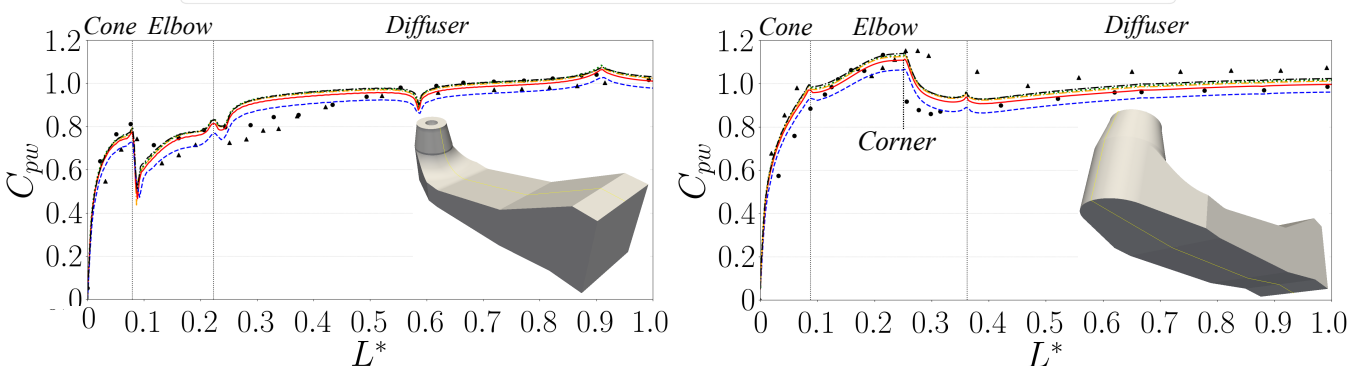

Figure 4: $C_{p w}(\mathrm{Eq} 14)$ distributions along the upper and lower wall centrelines using the cfMesh parameters shown in Table 3. Steady-state and time-averaged unsteady simulations. $L^{*}$ is the normalised length of the lower and upper walls along the centerline (-). 
Of the grids considered,'Mesh A', with the coarsest grid, showed the poorest consistency to the other grid resolutions around the lower floor of the heel section. This can be largely attributed to the limitations of the turbulence modelling in the near-wall region or lack of flow physics from the mesh resolution in the freestream. Čarija et al. 21] had previously demonstrated that the choice of turbulence model had little effect on the wall pressure, but did comment on the sensitivity of the near-wall resolution. Despite this result, the minimum number of cells required to adequately capture the complex flow along the draft tube walls (especially separation) was for 'Mesh B' or 'Mesh D'. Furthermore, to maintain a near-wall resolution range of $30<y_{1}^{+}<300$ for the first-cell height from the walls, required for the $k-\epsilon$ models, the boundary-layer parameters from 'Mesh B' or 'Mesh D' are required. Finally, it can also be observed that there is little deviation between the steady-state and unsteady (time-averaged) simulations.

\subsection{Validation of CFD modelling}

Table 4 shows the calculated performance quantities outlined in $\$ 2.2$ for different grid resolutions and those obtained from the literature. It can be seen that the present results largely agree with the equivalent CFD studies - especially those from the more recent papers $([6,7,28,8]$ ), which use a similar CFD setup to the present work. However, the benchmark experimental results for pressure recovery from the 2nd Turbine-99 Workshop [14 is generally larger than the CFD results. This observation is unsurprising, as $C_{p}$ is attained through an area-weighted averaging over the cross-section and is therefore more difficult to determine experimentally. For the experimental approximation of pressure recovery, $C_{p}$ (Exp.), the mean pressure at the outflow was estimated from the wall pressure, $p_{\text {out }, \text { wall }}$, since the pressure can only be measured in this vicinity at the outflow section [14]. The method of calculating $C_{p}$ (Exp.) has been replicated in the present CFD calculations, based on probe locations specified in the 2nd Turbine-99 workshop [14]. A distinctive $3-4 \%$ increase in pressure recovery is attained over the equivalent area-weighted results. Quantification of the alternate pressure recovery $C_{p}^{\prime}$ demonstrates that this is more sensitive to the grid resolution than the conventional $C_{p}$, due to the fluctuating velocity distribution at the sample plane.

Like $C_{p}, \zeta$ requires the measurement the flowfield over the inflow and outflow cross-sections and is seldom quantified in experimental work. However, for CFD it is easily determined. It can be seen in Table 4 that the validation 
of $\zeta$ becomes difficult due to the limited number of sources. The summary of CFD results from the Turbine-99 Workshops [17, 14, 18, shows a scatter of values for $\zeta_{2}$ in which the present results fall within this range. It can also be seen in Table 4 , for the present work, the values $\zeta_{1}$ and $\zeta_{2}$ increase with number of cells, while the values of $C_{p}$ decrease to a converged result.

\begin{tabular}{|c|c|c|c|c|c|}
\hline Case & $\begin{array}{c}C_{p} \\
(\mathrm{Eq}, 10)\end{array}$ & $\begin{array}{c}C_{p}^{\prime} \\
(\mathrm{Eq}, 13)\end{array}$ & $\begin{array}{c}C_{p} \\
\text { (Exp.) }\end{array}$ & $\begin{array}{c}\zeta_{1} \\
(\mathrm{Eq}, 11)\end{array}$ & $\begin{array}{c}\zeta_{2} \\
(\mathrm{Eq}, 12)\end{array}$ \\
\hline Mesh A & 0.9641 & 0.9655 & 0.9836 & 0.1375 & 0.1562 \\
\hline Mesh B & 0.9563 & 0.9586 & 0.9890 & 0.1445 & 0.1630 \\
\hline Mesh C & 0.9563 & 0.9580 & 0.9908 & 0.1463 & 0.1645 \\
\hline Mesh D & 0.9562 & 0.9571 & 0.9820 & 0.1465 & 0.1647 \\
\hline Mesh B (unsteady) & 0.9566 & 0.9559 & 0.9895 & 0.1447 & 0.1658 \\
\hline [14] Exp. & {$[-]$} & {$[-]$} & $1.02-1.1$ & {$[-]$} & $0.09 \pm 0.06$ \\
\hline [26] CFD (summary) & $0.887-0.991$ & {$[-]$} & {$[-]$} & {$[-]$} & $0.066-0.172$ \\
\hline 18] CFD (summary) & $0.710-1.032$ & {$[-]$} & {$[-]$} & {$[-]$} & $0.043-0.301$ \\
\hline [6] CFD (steady, $k-\epsilon$ ) & 0.9573 & {$[-]$} & {$[-]$} & {$[-]$} & 0.0790 \\
\hline [7] CFD (steady, $k-\epsilon$ ) & 0.9588 & {$[-]$} & {$[-]$} & {$[-]$} & {$[-]$} \\
\hline [7] CFD (unsteady, $k-\epsilon$ ) & 0.9588 & {$[-]$} & {$[-]$} & {$[-]$} & {$[-]$} \\
\hline [8] CFD (steady, $k-\epsilon$ ) & 0.8855 & {$[-]$} & {$[-]$} & 0.1755 & {$[-]$} \\
\hline
\end{tabular}

Table 4: Performance quantities obtained from the present grids, and those obtained from the literature. $C_{p}$ (Exp.) calculates the pressure at the inflow and outflow boundaries based on probe locations specified by the 2nd Turbine-99 workshop [14.

It is also interesting to observe the development of performance quantities along the draft tube. A series of sample planes are placed along the draft tube in the positions indicated in Fig 5 (top). The performance quantities were calculated on these planes using Eqs 10 and 12, where out is synonymous with the position of the plane (e.g., $p_{\text {out }}=p_{A}$ at position $A$ ). Fig. 5 (bottom) shows the development of the performance quantities along the draft tube for different grid resolutions. The $C_{p}$ progression conforms the observation above for its insensitivity to the grid resolution and use of steady/unsteady simulations. Furthermore, it can also be seen that the pressure recovery is largest within the inflow cone and heel regions. $\zeta$ on the other hand is considerably more sensitive to the grid resolutions than $C_{p}$, but appears insensitive to the use of steady/unsteady simulations. 


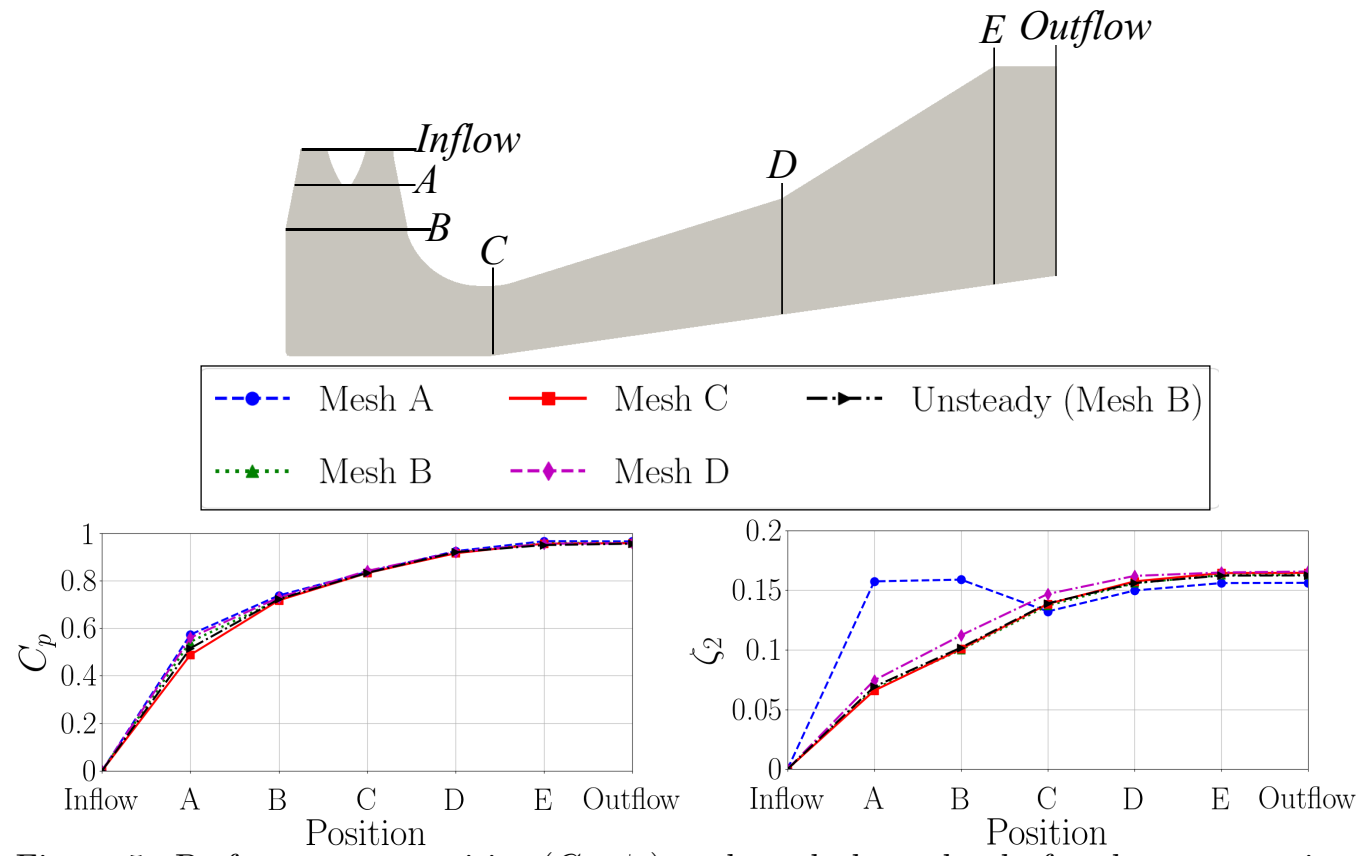

Figure 5: Performance quantities $\left(C_{p}, \zeta_{2}\right)$ evaluated along the draft tube cross-sections for various mesh resolutions.

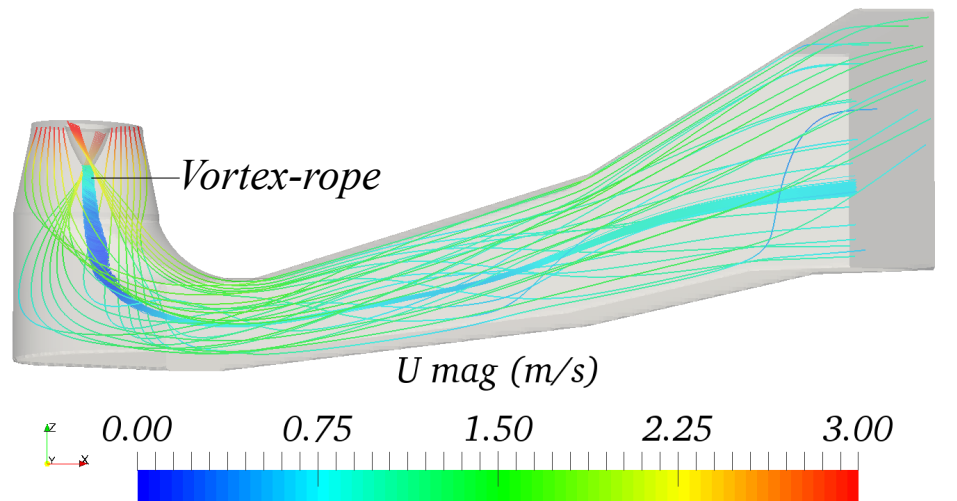

Figure 6: Streamlines of the flow along the base draft tube design.

Fig 6 shows the velocity streamlines along the draft tube geometry from the present work. Despite the disparity of reported pressure recovery, the vortex-rope formation is similar to those previously reported in equivalent CFD studies (e.g., [6, 21]) but not in experimental observations [31]. Considering the present CFD setup, there are three possible explanations for the differences to the experimental results: 
1. the flow through the draft tube is assumed to be at a steady-state, even though it clearly posses transient characteristics, leaving many of the impressionable flow features (i.e. extent of flow separation) absent;

2. the limitations of RANS modelling: in theory, increasing the fidelity of the turbulence modelling approach would result in a closer simulated flowfield to the equivalent experiments. However, according to the participants of the 2nd Turbine-99 Workshop [14], it is debated whether the standard $k-\epsilon$ model is capable of predicting the major flow features of the base case and performance quantities [17, 14];

3. the assumptions made in simulating the discharge from a Kaplan turbine. These are threefold: the reliability of the symmetrical axial, radial, and tangential velocity profiles suggested in the Turbine-99 workshops. Regarding the first assumption, the axial velocity profile is unlikely to be symmetric [32, forming a 'Rotating Vortex-Rope' below the runner, as observed in experiments [26]. Secondly, the radial velocity has a significant influence on the vortex-rope formation and draft tube efficiency [33]. The boundary condition for the radial velocity (Eqs.6.7) serves as an intuitive approximation. Finally, the tangential velocity requires a very fine grid resolution near the wall of the runner as the profile alternates in sign (large velocity gradient) in this region. This change of sign originates from the log-wall assumption and the fitting of measured tangential velocity profile [26], whose accuracy is questionable [20].

It is suggested that despite the limitations described above, the present CFD methodology provides a suitable approximation of the flowfield and draft tube performance values. The quantified wall pressures and performance quantities carried out in this section support this conclusion.

\subsection{Verification of numerical errors}

In this section, the method for estimating the uncertainty of CFD solution due to the resolution of the grid is outlined.

Grid independency analysis was conducted through the GCI (Grid Convergence Index) method [12, which has previously been employed for draft tube flows [34]. The representative cell size $h_{i}$ for each grid $i$ is described

$$
h_{i}=\left[\frac{1}{N_{i}} \sum_{j=1}^{N_{i}}\left(\Delta V_{j}\right)\right]^{1 / 3},
$$


where $N_{i}$ is the number of cells, and $V_{j}$ is the volume of each cell $j$. As observed in $\$ 2.4$. 'Mesh A' was unable to produce physically meaningful results due to the low resolution of the internal domain and near-wall regions. Therefore, grid resolutions 'Meshes B-D' outlined in \$2.3 were chosen for this analysis. The maximum non-orthogonality for the finest grid ('Mesh D') was approximately $60^{\circ}$, while the average value is approximately $7^{\circ}$. The resulting grid refinement factor $\left(h_{\text {coarse }} / h_{\text {fine }}\right)$ is 3.825 - larger than the minimum recommended 1.3 [12]. The three grids are ranked $h_{1}<h_{2}<h_{3}$. The apparent order of grid convergence, $\alpha$, is determined through a fixedpoint iteration of the expression:

$$
\alpha=\frac{1}{\ln \left(h_{2} / h_{1}\right)}|\ln | \frac{\phi_{3}-\phi_{2}}{\phi_{2}-\phi_{1}}\left|+\ln \left(\frac{\left(h_{2} / h_{1}\right)^{\alpha}-1 \cdot \operatorname{sgn}\left(\frac{\phi_{3}-\phi_{2}}{\phi_{2}-\phi_{1}}\right)}{\left(h_{3} / h_{2}\right)^{\alpha}-1 \cdot \operatorname{sgn}\left(\frac{\phi_{3}-\phi_{2}}{\phi_{2}-\phi_{1}}\right)}\right)\right|,
$$

where $\phi$ is the performance quantity under consideration. Hence, an extrapolated value for the performance quantity $\phi$ can be obtained using

$$
\phi_{e x t}^{21}=\frac{\left(h_{2} / h_{1}\right)^{\alpha} \phi_{1}-\phi_{2}}{\left(h_{2} / h_{1}\right)^{\alpha}-1}
$$

and the grid uncertainty estimations are determined:

Approximate relative error,

$$
e_{a}^{21}(\%)=100 \cdot\left|\frac{\phi_{1}-\phi_{2}}{\phi_{1}}\right| ;
$$

extrapolated relative error,

$$
e_{e x t}^{21}(\%)=100 \cdot\left|\frac{\phi_{e x t}^{21}-\phi_{1}}{\phi_{e x t}^{21}}\right| ;
$$

fine-grid convergence index,

$$
G C I_{\text {fine }}^{21}(\%)=100 \cdot\left(\frac{1.25 e_{a}^{21}}{\left(h_{2} / h_{1}\right)^{\alpha}-1}\right) .
$$

The pressure recovery factor $(\mathrm{Eq}, 10)$ was used to assess the grid uncertainty. It should be noted this is estimated through an area-weighted process - reducing the sensitivity to the grid. To demonstrate this aspect, an arithmetic 
average of the pressure recovery is performed over the faces of each sample plane (see Fig.5(top):

$$
C_{p}\left(\sum\right)=\frac{\frac{\sum_{j=1}^{N_{\text {out }}} p_{\text {out }}}{N_{\text {out }}}-\frac{\sum_{j=1}^{N_{\text {in }}} p_{\text {in }}}{N_{\text {in }}}}{\frac{1}{2} \rho\left(\frac{Q}{A_{\text {in }}}\right)^{2}} .
$$

Using this definition, the GCI results are shown in Table 5. It can be seen that the apparent order of convergence is limited to the order of the numerical method (2nd). Naturally, some numerical diffusion is expected, with the estimation being suitably larger than 1 [12] for all cross-sections along the draft tube. Moreover, the estimated uncertainty reduces monotonically along the draft tube - regardless of the local flowfield features. The largest uncertainty is $4.76 \%$ at the base of the runner hub, which is still sufficient for interpretation $(<10 \%[12])$.

\begin{tabular}{|c|c|c|c|c|c|c|}
\hline$\phi$ & Plane & $\alpha$ & $\phi_{\text {ext }}^{21}$ & $e_{a}^{21}(\%)$ & $e_{\text {ext }}^{21}(\%)$ & $G C I_{\text {fine }}^{21}(\%)$ \\
\hline \hline \multirow{4}{*}{$C_{p}$} & $\mathrm{~A}$ & 1.2235 & 0.3071 & -1.9214 & -4.3118 & -4.7660 \\
$\left(\sum\right)$ & $\mathrm{B}$ & 1.3129 & 0.6274 & -1.8089 & -1.8354 & -1.9385 \\
& $\mathrm{C}$ & 1.5317 & 0.8264 & -0.8236 & -1.2972 & -1.6010 \\
& $\mathrm{D}$ & 1.6439 & 0.8929 & -0.6633 & -1.2289 & -1.2797 \\
& $\mathrm{E}$ & 1.7604 & 0.9561 & -0.7958 & -0.7758 & -0.9623 \\
& Outflow & 1.8814 & 0.9569 & -0.6191 & -0.3188 & -0.3973 \\
\hline
\end{tabular}

Table 5: GCI results for the un-weighed averaging for the pressure recovery (Eq21) at sample planes along the base geometry (see Fig 5 (top)).

Table 6 shows the GCI results for the area-weighted estimation of the pressure recovery $(\mathrm{Eq} 10)$. It can be seen that this representation shows a greater independence to the grid resolution than the arithmetic estimation (Eq21). At the same time, it can be seen that the apparent order of convergence (and corresponding uncertainty) now fluctuates with the local cross-sectional area of the sample plane. It should be noted that the values of extrapolated pressure recovery are similar regardless of the estimation method. 


\begin{tabular}{|c|c|c|c|c|c|c|}
\hline$\phi$ & Position & $\alpha$ & $\phi_{\text {ext }}^{21}$ & $e_{a}^{21}(\%)$ & $e_{\text {ext }}^{21}(\%)$ & $G C I_{\text {fine }}^{21}(\%)$ \\
\hline \hline \multirow{6}{*}{$C_{p}$} & $\mathrm{~A}$ & 2.5424 & 0.5319 & -0.4539 & -0.5803 & -0.7212 \\
& $\mathrm{~B}$ & 2.1475 & 0.7349 & -0.1050 & -0.1662 & -0.2074 \\
& $\mathrm{C}$ & 5.7901 & 0.8380 & -0.0086 & -0.0031 & -0.0039 \\
& $\mathrm{D}$ & 4.4686 & 0.9231 & -0.0869 & -0.0490 & -0.0612 \\
& $\mathrm{E}$ & 3.8923 & 0.9563 & -0.1761 & -0.1232 & -0.1538 \\
& Outflow & 3.3593 & 0.9562 & -0.3801 & -0.3312 & -0.4178 \\
\hline
\end{tabular}

Table 6: GCI results for the area-weighted averaging for the pressure recovery (Eq10 at sample planes along the base geometry (see Fig 5 (top)).

\section{Draft tube design study}

In this section, the CFD methodology described in $\$ 2.1$ is used to evaluate proposed design recommendations for the draft tube in the literature. The focus of this analysis will be on the inflow cone and outer-heel, as the greatest pressure recovery occurs these regions. The automatic construction of the closed-manifold surfaces was achieved using Glyph scripting (using TCL) in Pointwise R18.2. These were imported to cfMesh which automatically generated the CFD grid for each draft tube design (described in $\$ 2.3$ ).

\subsection{Inflow cone section}

As seen in Fig.5, the greatest recovery of pressure occurs in the inflow cone, due to flow separation below the runner hub. This phenomenon is controlled to some extent by the runner hub design (diameter, length, and shape of bulb). While altering the shape of the runner hub is not considered in this research, the same effect can be achieved by altering the cross-sectional area surrounding this component [6, 35]. Convex and concave inflow cone designs are considered in the present work, along with the optimum design from 2nd part of this research [36] - which has a slighter larger radius than the base geometry.

To alter the inflow cone radius, a single control point is positioned at the lowest level of the hub. The side of the inflow cone was represented by a single Catmull-Rom spline [37] — possessing $C^{1}$ parametric continuity. The spline implementation is indicated in Fig.8(a). The considered radii of the inflow cones were $r=0.3 m, 0.205 m$, and $0.5 m$ (base design, $0.28 m$ ) - the last two cases are shown in Fig.8(b) and (c). 


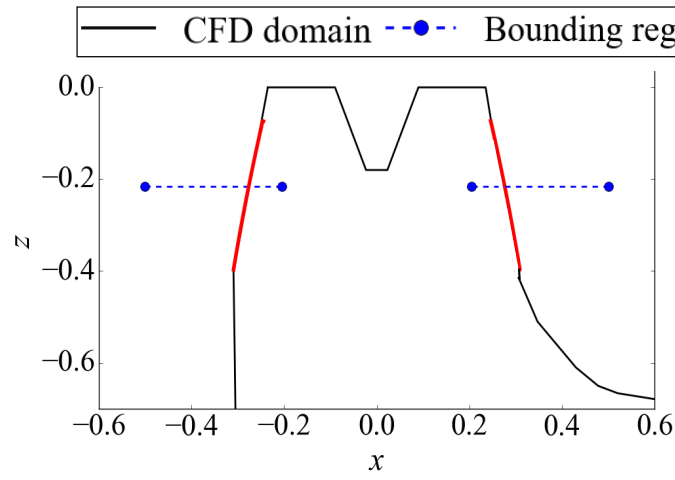

(a)

\section{Catmull-Rom spline}

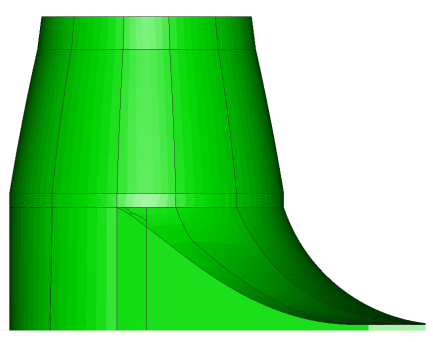

(b)

Figure 7: A demonstration of the inflow cone radius bounds considered in this work; (a) a schematic of the inflow cone with the bounds for the control point; (b) the base design. All dimensions are in $\mathrm{cm}$.

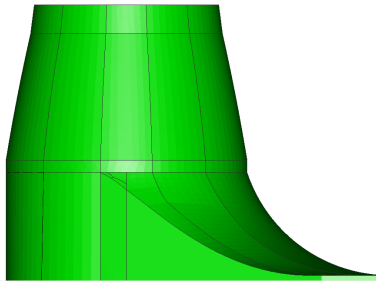

(a)

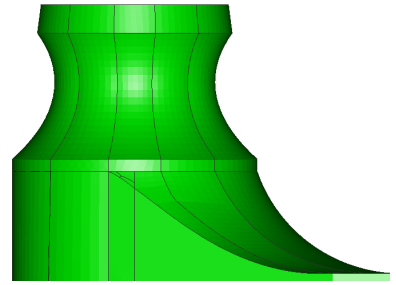

(b)

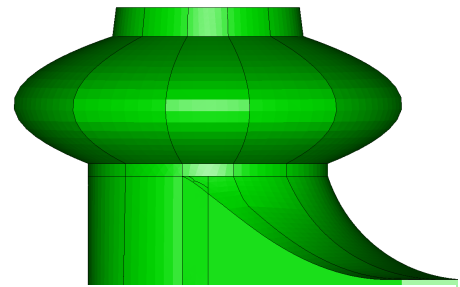

(c)

Figure 8: A demonstration of the inflow cone geometries considered investigation; (a) the base design; (c) the smallest radius considered; (d) the largest radius considered.

Fig, 9 shows the velocity streamlines through the draft tube with different inflow cone radii. It can be seen that the vortex-rope dissipates (along with the swirl intensity) as the area around the runner hub is reduced. For the convex design, the effective vortex cavities cause the flow to separate along the inflow cone walls, though the vortex rope is largely left unaffected by this effect. This trend confirms the speculations made by several authors [1, 26, 35, 38]. 

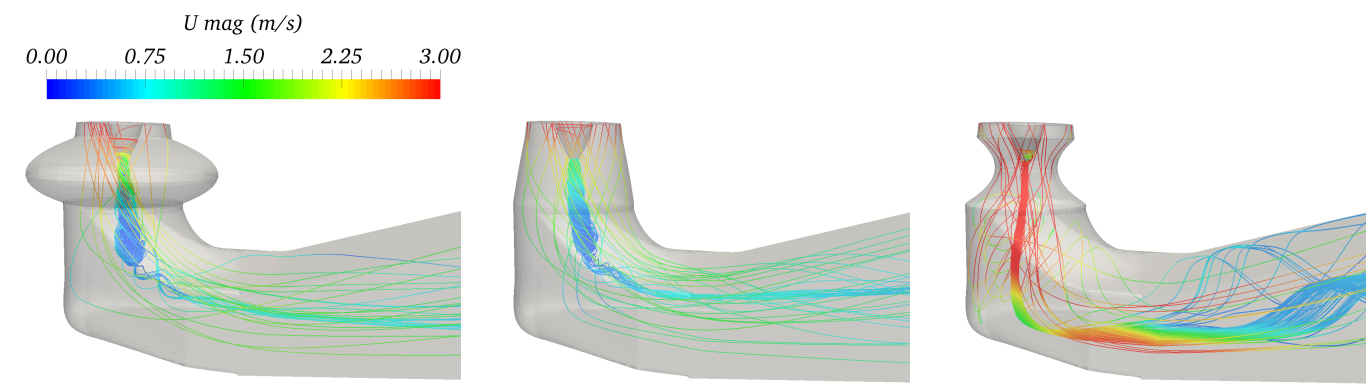

Figure 9: Streamlines along the draft tube with various inflow cone designs (with base heel and diffuser).

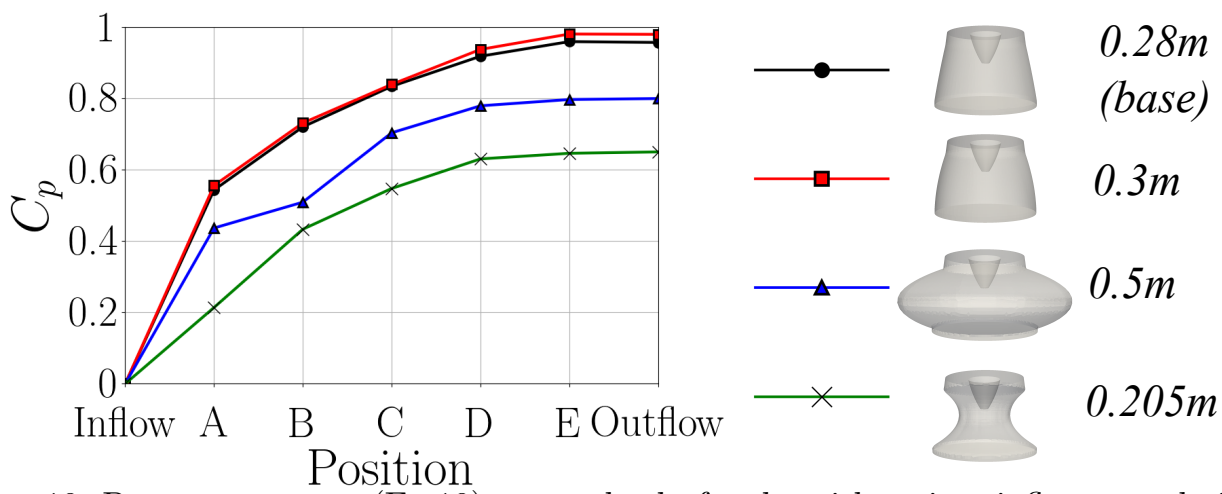

Figure 10: Pressure recovery (Eq 10 across the draft tube with various inflow cone designs.

Fig 10 shows the progression of pressure recovery along the draft tube for various inflow cone designs. The location of the sample planes are indicated in Fig.5(top). From Fig.10, a number of trends can be observed:

- the pressure recovery along the heel and diffuser sections are scaled according to the pressure recovery around the inflow cone (sampleplane 'A');

- The extreme designs of large and small cone radii have a detrimental effect to the overall pressure recovery;

- For the large cone radius, the pressure recovery reduced around the base of the cone (sample-plane 'B').

Overall, it is shown in this section that the design of the inflow cone significantly affects the vortex-rope and resulting efficiency of the draft tube. GCI 
analysis (see $\$ 2.5$ ) is also applied to the draft tube designs. Input parameters for 'Meshes B-D' (\$2.3) were used to generate the grids while the pressure recovery factor (Eq.10) was used to assess the grid uncertainty. The results of this analysis are shown in Table 7, with the apparent trends:

- inflow cone with radius $0.3 \mathrm{~m}$ has similar results to the base design (Table 6);

- reducing the radius of the inflow cone increases the error significantly, with no apparent relation to the local cross-sectional areas;

- the inflow cone with the largest radius has a similar pattern to the base design but with larger errors.

\begin{tabular}{|c|c|c|c|c|c|c|}
\hline Inflow cone & Plane & $\alpha$ & $\phi_{\text {ext }}^{21}$ & $e_{a}^{21}(\%)$ & $e_{\text {ext }}^{21}(\%)$ & $G C I_{\text {fine }}^{21}(\%)$ \\
\hline \hline \multirow{5}{*}{$0.205 m$} & $\mathrm{~A}$ & 5.4742 & 0.2130 & -1.2257 & -0.3222 & -0.4014 \\
& $\mathrm{~B}$ & 2.7336 & 0.4105 & -0.6188 & -0.5474 & -0.6487 \\
& $\mathrm{C}$ & 5.1024 & 0.5263 & -0.5464 & -3.9515 & -0.4752 \\
& $\mathrm{D}$ & 2.1918 & 0.6241 & -0.8178 & -0.9418 & -0.1166 \\
& $\mathrm{E}$ & 3.2586 & 0.6237 & -0.5270 & -0.3521 & -0.4252 \\
& Outflow & 4.6411 & 0.6482 & -0.1270 & -0.2220 & -0.2769 \\
\hline \multirow{5}{*}{$0.3 m$} & $\mathrm{~A}$ & 2.7268 & 0.5540 & -4.5258 & -3.9564 & -4.7572 \\
& $\mathrm{~B}$ & 2.7812 & 0.7390 & -0.3023 & -0.2477 & -0.3088 \\
& $\mathrm{C}$ & 5.0627 & 0.8382 & -0.2432 & -0.0741 & -0.0926 \\
& $\mathrm{D}$ & 4.6212 & 0.9357 & -0.3263 & -0.1178 & -0.1471 \\
& $\mathrm{E}$ & 3.6197 & 0.9776 & -0.3351 & -0.1835 & -0.2290 \\
& Outflow & 2.8871 & 0.9763 & -0.3275 & -0.2540 & -0.3167 \\
\hline \multirow{5}{*}{$0.5 m$} & $\mathrm{~A}$ & 1.4943 & 0.3938 & -2.8463 & -5.6171 & -6.6480 \\
& $\mathrm{~B}$ & 2.0309 & 0.4924 & -2.2497 & -1.0436 & -1.2910 \\
& $\mathrm{C}$ & 4.6430 & 0.7323 & 1.4614 & 0.5213 & 0.6551 \\
& $\mathrm{D}$ & 2.4561 & 0.8021 & 1.3459 & 1.2986 & 1.6446 \\
& $\mathrm{E}$ & 1.9325 & 0.8340 & 1.4648 & 1.9388 & 2.4714 \\
& Outflow & 1.3050 & 0.8297 & 1.0744 & 2.3114 & 2.9577 \\
\hline
\end{tabular}

Table 7: GCI results for the area-weighted averaging for the pressure recovery (Eq, 10 at sample planes (see Fig,5) along geometries with different inflow cone radii. 


\subsection{Elbow section}

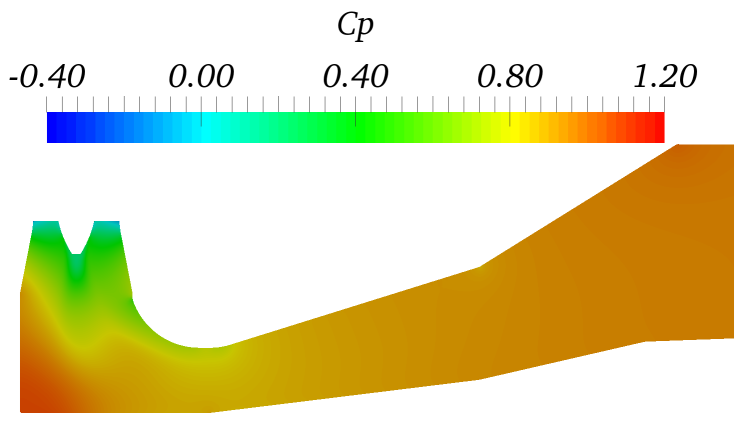

Figure 11: Pressure recovery (Eq.10 contour down the centerline for base design.

The sharp-heel construction of the base design is a rather unusual choice from the perspective of the fluid flow. Indeed, the presence of a sharp-heel is reported to contribute an efficiency loss $\left(C_{p}\right)$ of approximately $0.3-2.3 \%$ [1. As seen in Fig.11, a significant variation of pressure can be seen in the elbow as the flow is redirected from the inflow cone to the diffuser. The stagnation region creates a diversion of the flow to the outer-wall of the elbow, forming a non-uniform velocity distribution at the opening of the diffuser section. At the same time, the sudden changes in cross-sectional area along the elbow incurs large regions of flow separation, reducing the draft tube efficiency. Based on these characteristics, the draft tube can be improved by maintaining or reducing the cross-sectional areas across the elbow section, or by incorporating design features which mitigate flow separation.

Along with the base (sharp-heel) design, this section will analyse the draft tube with the following outer-heel designs:

1. curved-heel proposed by Dahlbäck [39];

2. expanded-heel (vortex-chamber) inspired by [40, 41, 42];

3. chamfered-heel proposed by Daniels et al. [36].

A flexible method was chosen to create the heel shapes described above. A Catmull-Rom spline was implemented on the $x z$-center-plane on the outerwall of the heel, as indicated in Fig.13a, which is subsequently projected around the heel as indicated in Fig 13b. Fig, 12 shows the schematic of the Catmull-Rom spline implementation. The proposed representation is also capable of recreating the original sharp-heel design. 


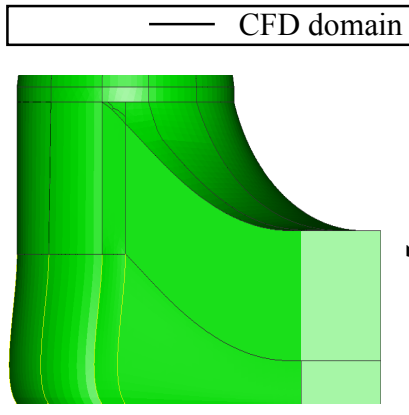

(a)

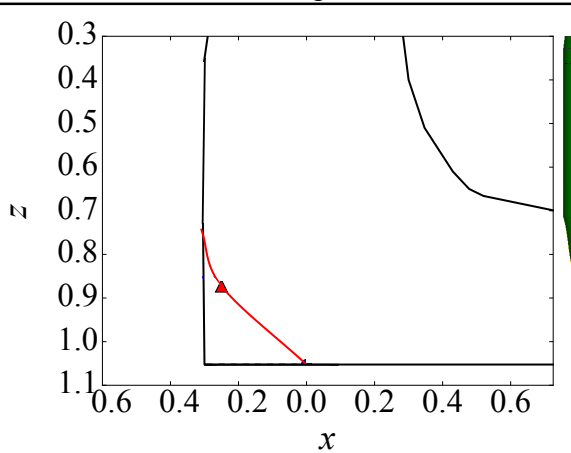

(b)
Catmull-Rom spline

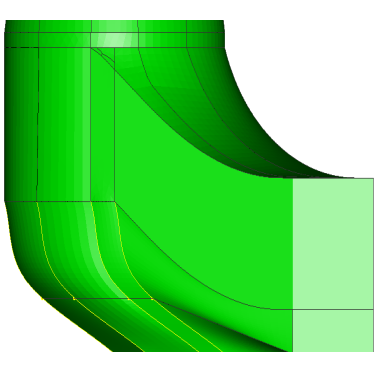

(c)

Figure 12: A demonstration of altering the heel design; (a) base heel construction using proposed heel representation; (b) schematic of the Catmull-Rom spline implementation, and control point; (c) a demonstration of the deformed heel using the spline formation in (b). All dimensions are in $\mathrm{cm}$.

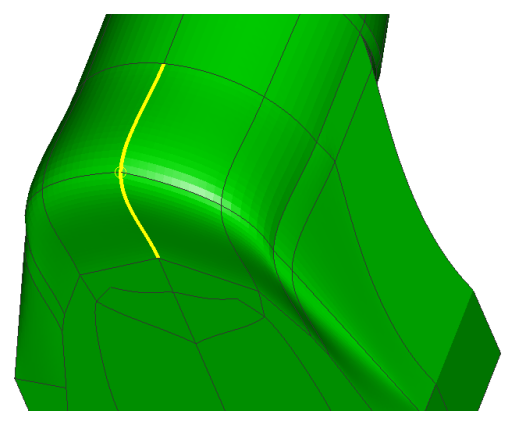

(a) Construction of the Catmul-Rom spline (yellow) on the heel of the draft tube.

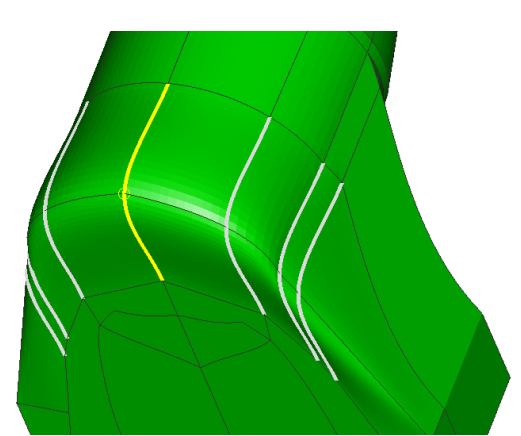

(b) Projection of the Catmul-Rom spline on the remaining vertices of the heel.

Figure 13: Implementation of the Catmull-Rom spline on the heel section.

Fig 14 shows the normalised pressure and velocity contours along the $x z$ center-plane for the sharp, curved, chamfered, and expanded heel designs. For the sharp-heel, the flowfield shows three separation regions: beneath the runner cone, outer corner of the heel, and upper wall at the entrance of the diffuser. When considering the curved-heel design, the recirculation in the heel corner disappears, increasing the pressure recovery by $1.92 \%$ to the sharp-heel design; this estimation is slightly larger than the experimental prediction of $1-1.5 \%$ [39]. A similar phenomenon can be seen for the expanded-heel, with a $1 \%$ increase of pressure recovery to the sharp-heel design. Finally, for the chamfered heel, small separation regions are formed at 
the top-left and bottom walls. The pressure recovery increases by $2.79 \%$ to the sharp-heel design. Furthermore, it can be seen in Fig.14 that the pressure flowfield around the inner-wall of the heel is largely insensitive to the heel design. The noticeable difference between the draft tube designs can be seen for the separation region below the runner hub. The velocity contours show the recirculation in this region increases with the expansion of the heel. Hence, a larger separation region beneath the runner hub is created reducing the pressure recovery. Smoothing the sharp-heel corner with an curved (or chamfered) heel reduces the swirl intensity of the flow and increases axial velocity across the inflow cone and heel, which consequently increases the draft tube efficiency.

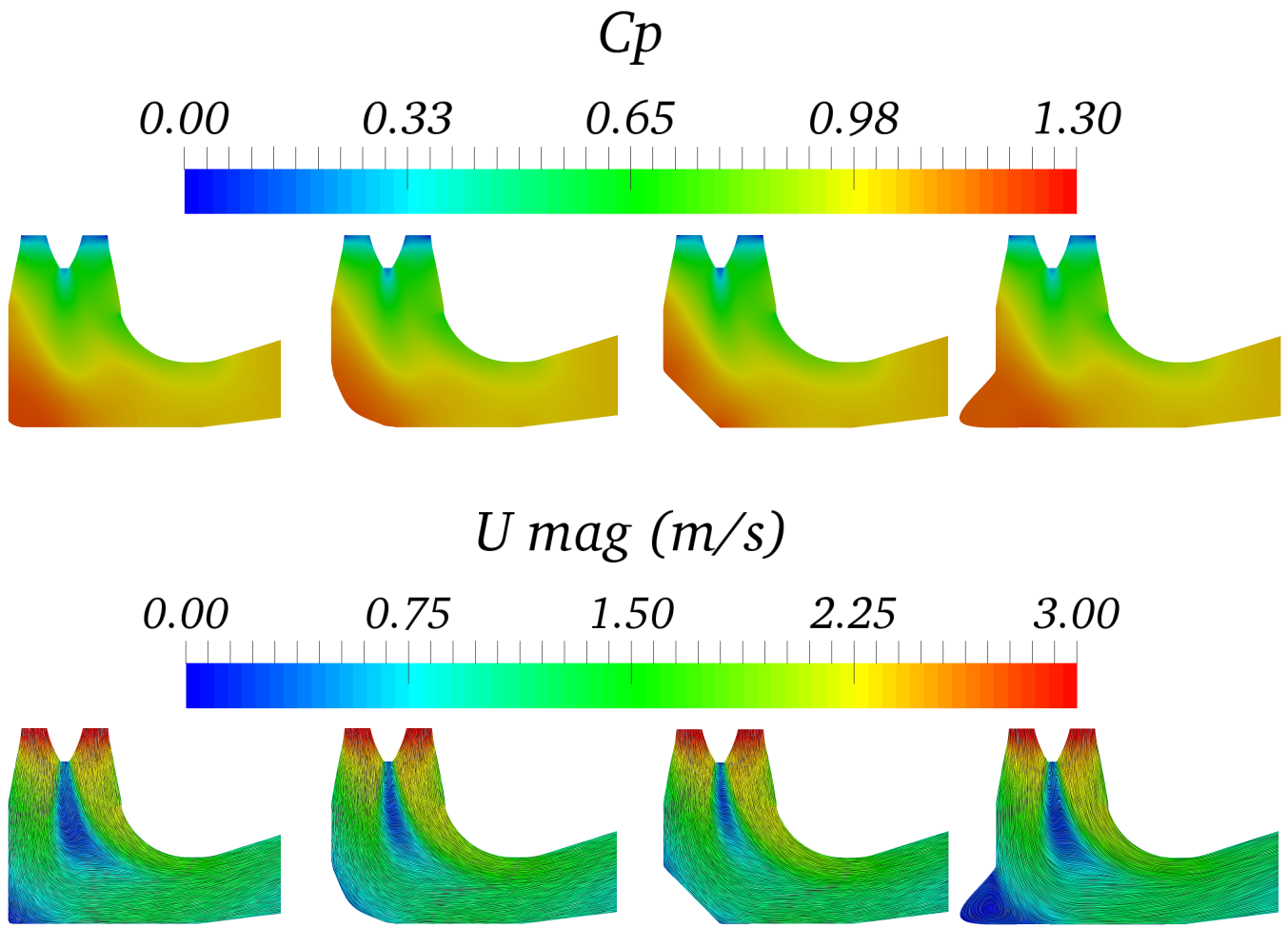

Figure 14: The normalised pressure distribution (top) and velocity magnitude (bottom) along the $x z$-center-plane through the draft tube. From left-to-right: base geometry, curved-heel [39], chamfered [36], and expanded heel design.

Fig, 15 shows the pressure recovery across various sample-planes (see Fig.5(top)) along the draft tube for the various heel designs. It can be seen that regardless of the heel design, the pressure recovery remains unperturbed in the inflow 
cone and heel sections of the draft tube. The difference in pressure recovery occurs in the diffuser section - downstream of the heel. Hence, it can be deduced that the heel design has a significant effect on the separation region below the runner hub, which, while the pressure field is relatively unchanged in the inflow cone and heel section, affects the uniformity of the velocity at the entrance of the diffuser section and pressure recovery downstream of the heel.

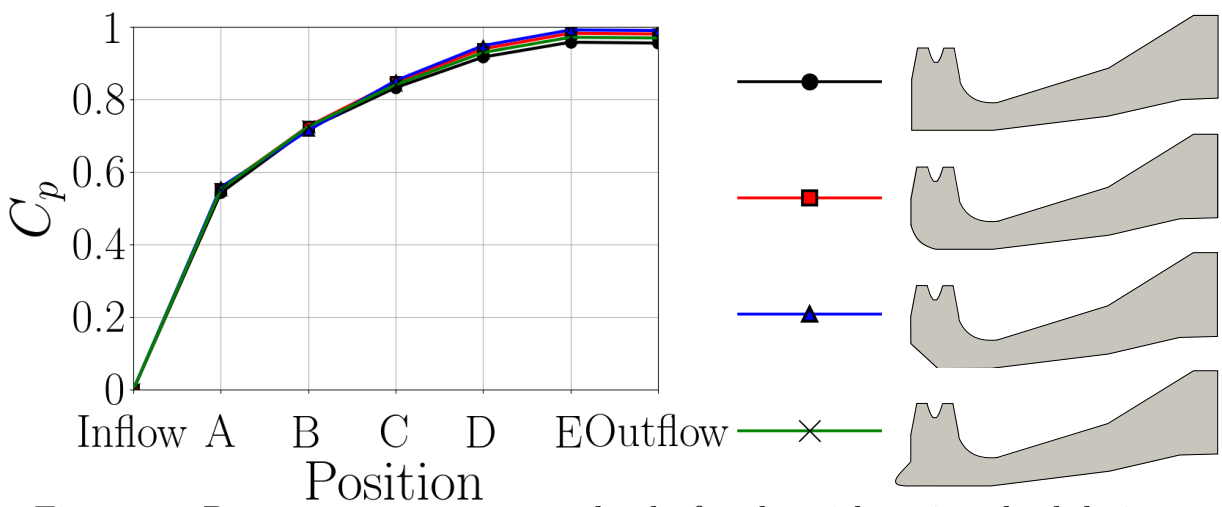

Figure 15: Pressure recovery across the draft tube with various heel designs.

Finally, GCI analysis (see $\$ 2.5$ ) is applied to the draft tube designs. Input parameters for 'Meshes B-D' (Table 3) were used to generate the grids in each design, while the pressure recovery factor $(\mathrm{Eq}, 10)$ was used to assess the grid uncertainty. The results from this are shown in Table 8. Again, like the base design, it can be seen that the uncertainty fluctuates with the local crosssectional area. At the same time, the grid uncertainty is considerably smaller than the maximum allowed $(<10 \%$ [12]) thus demonstrating the robustness of the grid regeneration method and methodology for estimating the pressure recovery. 


\begin{tabular}{|c|c|c|c|c|c|c|}
\hline Heel design & Plane & $\alpha$ & $\phi_{\text {ext }}^{21}$ & $e_{a}^{21}(\%)$ & $e_{\text {ext }}^{21}(\%)$ & $G C I_{\text {fine }}^{21}(\%)$ \\
\hline \hline \multirow{5}{*}{ Curved } & $\mathrm{A}$ & 2.4108 & 0.5433 & -0.0407 & -0.0408 & -0.0510 \\
& $\mathrm{~B}$ & 2.0618 & 0.7315 & -0.3570 & -0.4449 & -0.5536 \\
& $\mathrm{C}$ & 5.1458 & 0.8437 & -0.1634 & -0.0485 & -0.0606 \\
& $\mathrm{D}$ & 3.4611 & 0.9347 & -0.2233 & -0.1318 & -0.1645 \\
& $\mathrm{E}$ & 2.9682 & 0.9773 & -0.2381 & -0.1671 & -0.2085 \\
& Outflow & 2.4595 & 0.9753 & -0.2147 & -0.2101 & -0.2620 \\
\hline \multirow{5}{*}{ Expanded } & $\mathrm{A}$ & 2.3512 & 0.5451 & -0.1761 & -0.1867 & -0.2291 \\
& $\mathrm{~B}$ & 2.0485 & 0.7247 & -0.1156 & -0.1451 & -0.1811 \\
& $\mathrm{C}$ & 5.6878 & 0.8375 & -0.1463 & -0.0357 & -0.0446 \\
& $\mathrm{D}$ & 4.4210 & 0.9260 & -0.1643 & -0.0645 & -0.0806 \\
& $\mathrm{E}$ & 3.6479 & 0.9675 & -0.1839 & -0.1012 & -0.1264 \\
& Outflow & 3.2176 & 0.9661 & -0.1643 & -0.1792 & -0.1514 \\
\hline \multirow{5}{*}{ Chamfered } & $\mathrm{A}$ & 2.6511 & 0.5441 & -0.0983 & -0.0866 & -0.1081 \\
& $\mathrm{~B}$ & 2.0793 & 0.7265 & -0.1369 & -0.1684 & -0.2101 \\
& $\mathrm{C}$ & 5.1780 & 0.8486 & -0.1653 & -0.0403 & -0.0503 \\
& $\mathrm{D}$ & 2.9135 & 0.9425 & -0.2562 & -0.1967 & -0.2454 \\
& $\mathrm{E}$ & 2.8862 & 0.9858 & -0.2443 & -0.1902 & -0.2373 \\
& Outflow & 2.4121 & 0.9840 & -0.2395 & -0.2409 & -0.3004 \\
\hline
\end{tabular}

Table 8: GCI results for the area-weighted averaging for the pressure recovery (Eq, 10) at sample planes (see Fig, 5) along geometries with different heel designs.

\section{Conclusions and future work}

An investigation into the numerical modelling of a number of elbow-type draft tube designs was carried out, focusing on the grid sensitivity and performance of each design. To achieve this, Computational Fluid Dynamics (CFD) was used to evaluate the performance of the given draft tube design, while the open-source meshing software ' $c f M e s h$ ' was used to automatically construct a predominately uniform hexahedral grid in each geometry.

A validation study of the numerical setup was undertaken on the sharpheeled Hölleforsen-Kaplan draft tube (base design). From this it was concluded that the steady-state assumption validated well with the equivalent experimental data. Moreover, the sensitivity of the draft tube performance measures to the CFD grid shows that the energy loss factor, $\zeta$, is considerably more sensitive than the pressure recovery factor $C_{p}$. It was also found that 
the estimation of pressure recovery through experimental measurements was consistently higher than the equivalent CFD method. The inflow cone and heel sections of the draft tube were identified as being the major contributing regions to the pressure recovery. Grid Convergence Index (GCI) analysis [12] was used to assess the uncertainty of pressure recovery related to the grid resolution. This was assessed at various cross-sections along the draft tube. From this two trends were identified:

1. estimating the pressure recovery by arithmetic averaging across the faces causes the apparent order of grid convergence to increase along the draft tube - limiting this to the order of numerical discretisation;

2. estimating the pressure recovery through area-weighted averaging caused the apparent order of grid convergence to fluctuate with the local crosssectional area - the associated uncertainty is significantly reduced.

The 2nd part of this paper focuses on assessing the draft tube performance with different inflow cone and heel designs proposed in the literature. Specifically, this work considered:

- Varying the radius of the inflow cone from a concave to conex shape, including the optimum design identified in Part-2 of this research [36];

- Curved [39], chamfered [36], and expanded [40, 41, 42] outer-heel designs.

Catmull-Rom splines were used to achieve the above geometries. It was found that the optimum inflow design [36] improved the pressure recovery by $2.79 \%$ to the base geometry. Significantly reducing and expanding the inflow cone radius reduced the efficiency by $30.79 \%$ and $13.5 \%$ respectively. Furthermore, changing the outer-heel to a design other than a sharp-heel increased the pressure recovery, with improvements: chamfered - $2.79 \%$, curved $-1.92 \%$, and expanded - 1\%. GCI analysis of the heel designs showed similar uncertainty values to the base design. On the other hand, for the various inflow cone designs, the apparent order of convergence for the concave design broke down along with the vortex-rope. For all geometries considered in this work, the grid uncertainty was less than 10\% (a limit specified by [12]) demonstrating the robustness of the automated meshing software.

Overall, the novel aspects of this paper include:

- a proposed method for the automated reconstruction of the geometry and CFD grid for each evaluation; 
- the characteristics of pressure recovery along the draft tube design through different methods of estimation;

- a study of the contributions of the inflow cone and heel components on the draft tube efficiency.

\subsection{Future work}

This work naturally leads to the following topics of investigation on draft tube design:

1. additional design considerations such as the turbine design, and robustness of the draft tube performance;

2. design evaluation of the runner hub geometry - providing a greater potential for pressure recovery and geometric flexibility than the inflow cone.

\section{Acknowledgments}

This work was supported by the UK Engineering and Physical Sciences Research Council [grant number EP/M017915/1]. The authors would also like to thank the helpful contributions of J. Angus, P. Burns, T. Dye, R. Gilbert, J. Gowans, S. Hardy, S. Hutchings and C. Ng. The CFD simulations were performed on the ISCA HPC in the Advanced Computing Facility in the University of Exeter, UK.

\section{References}

[1] M. Gubin, Draft tubes of Hydro-electric Stations, Amerind Publishing Co., New Dehli, 1973.

[2] B. Fabritius, G. Tabor, Improving the quality of finite volume meshes through genetic optimisation, Eng. Comp. 32 (3) (2016) 425-440. doi: $10.1007 / \mathrm{s} 00366-015-0423-0$.

[3] J. Samareh, Geometry and grid/mesh generation issues for CFD and CSM shape optimization, Optimization and Engineering 6 (1) (2005) 21-32. doi:10.1023/B:OPTE.0000048535.08259.a8. 
[4] T. Arispe, W. de Oliveira, R. Ramirez, Francis turbine draft tube parameterization and analysis of performance characteristics using CFD techniques, Renew. Energy 127 (2018) 114-124. doi:10.1016/j.renene. 2018.04.055.

[5] S. Daniels, A. Rahat, G. Tabor, J. Fieldsend, R. Everson, A review of shape distortion methods available in the openfoam framework for automated design optimisation, in: OpenFOAM: Selected Papers of the 11th Workshop, Springer International Publishing, 2019. doi:10.1007/ 978-3-319-60846-4_28.

[6] B. D. Marjavaara, T. S. Lundström, Redesign of a sharp heel draft tube by a validated CFD-optimization, Int. J. Numer. Methods Fluids 50 (8) (2006) 911-924. doi:10.1002/fld.1085.

[7] J. Hellström, B. Marjavaara, T. Lundström, Parallel CFD simulations of an original and redesigned hydraulic turbine draft tube, Adv. Eng. Softw. 38 (5) (2007) 338-344. doi:10.1016/j.advengsoft.2006.08. 013 .

[8] S. Galván, C. Rubio, J. Pacheco, S. Gildardo, C. Georgina, Optimization methodology assessment for the inlet velocity profile of a hydraulic turbine draft tube: part II - performance evaluation of draft tube model, J. Glob. Optim. 55 (4) (2013) 729-749. doi:10.1007/ s10898-012-0011-4.

[9] J. Bergström, Turbulence modeling and numerical accuracy for the simulation of the flow field in a curved channel, in: Proceedings, 1997 ASME Fluid Engineering Division Summer Meeting : FEDSM97-3298, Symposium on Separated and Complex Flows, 1997.

[10] B. J., R. Gebart, Estimation of numerical accuracy for the flow field in a draft tube, Int. J. Num. Meth. Heat \& Fluid Flow 9 (4) (1999) 472-487. doi:10.1108/09615539910266620.

[11] I. Celik, W.-M. Zhang, Calculation of numerical uncertainty using richardson extrapolation: application to some simple turbulent flow calculations, J. Fluids Eng. 117 (3) (1995) 439-445.

[12] I. Celik, U. Ghia, P. Roache, C. Freitas, H. Coleman, P. Raad, Procedure for estimation and reporting of uncertainty due to discretization in CFD 
applications, J. Fluids Eng. 130 (7) (2008) 078001-078001-4. doi:10. $1115 / 1.2960953$.

[13] A. Abbas, A. Kumar, Development of draft tube in hydro-turbine: a review, Int. J. Amb. Ener. 38 (3) (2017) 323-330. doi:10.1080/ 01430750.2015 .1111845 .

[14] T. Engström, L. Gustavsson, R. Karlsson (Eds.), Proceedings of Turbine-99 - Workshop II, Luleå University of Technology, Sweden, 2001.

[15] M. Cervantes, M. Lövgren, Radial velocity at the inlet of the turbine99 draft tube, in: Proceedings of the 2nd IAHR International Meeting of the Workgroup on Cavitation and Dynamic Problems in Hydraulic Machinery and Systems, Timisoara, Romania, October 24-26, 2007, no. $52(66)$ in Transaction of Mechanics, Scientific Bulletin of the Politehnica University of Timisoara, Romania, 2007, pp. 137-144.

[16] D. Marjavaara, S. Lundström, Response surface-based shape optimization of a francis draft tube, Int. J. Numer. Meth. Heat Fluid Flow 17 (1) (2007) 34-45. doi:10.1108/09615530710716072.

[17] B. R. Gebart, L. H. Gustavsson, R. I. Karlsson (Eds.), Turbine-99: workshop on draft tube flow, Luleå University of Technology, Sweden, 2000.

[18] M. Cervantes, T. Engström, L. Gustavsson, Proceedings of turbine-99 III: Workshop on draft tube flow, Luleå University of Technology (2005).

[19] N. Tanase, B. Florentina, G. Ciocan, Numerical simulation of the flow in the draft tube of the Kaplan turbine, U.P.B. Sci. Bull., Series D 74 (1) (2012) 83-90.

[20] H. Nilsson, M. Cervantes, Effects of inlet boundary conditions, on the computed flow in the turbine-99 draft tube, using OpenFOAM and CFX, IOP Conference Series: Earth and Environmental Science 15 (3) (2012) 032002. doi:10.1088/1755-1315/15/3/032002.

[21] Z. Čarija, Z. Mrša, L. Dragović, Turbulent flow simulation in kaplan draft tube, in: 5th International Congress of Croatian Society of Mechanics, 2006. 
[22] S. Galván, M. Reggio, F. Guibault, Assessment study of k-e turbulence models and near-wall modeling for steady state swirling flow analysis in draft tube using fluent, Eng. Appl. Comput. Fluid Mech. 5 (4) (2011) 459-478. doi:10.1080/19942060.2011.11015386.

[23] H. G. Weller, G. Tabor, H. Jasak, C. Fureby, A tensorial approach to computational continuum mechanics using object-oriented techniques, J. Comput. Phys. 12 (6) (1998) 620-631. doi:10.1063/1.168744.

[24] R. Issa, Solution of the implicitly discretised fluid flow equations by operator-splitting, J. Comput. Phys. 62 (1) (1986) 40-65. doi:10.1016/ 0021-9991(86)90099-9.

[25] S. Patankar, D. Spalding, A calculation procedure for heat, mass and momentum transfer in three-dimensional parabolic flows, Int. J. Heat Mass Transf. 15 (10) (1972) 1787-1806. doi:10.1016/ B978-0-08-030937-8.50013-1.

[26] U. Andersson, F. Engström, H. Gustavsson, R. Karlsson, The turbine-99 workshops - conclusions and recommendations, in: 22nd IAHR Symposium on Hydraulic Machinery and Systems, Stockholm, Sweden, June 29 - July 2, 2004, IAHR, 2004.

[27] C. Michael, W. Torsten, Special interest group on quality and trust in industrial CFD. Best Practice Guidelines, European Research Community on Flow, Turbulence and Combustion (ERCOFTAC), 2000.

[28] S. Galván, C. Rubio, J. Pacheco, C. Mendoza, M. Toledo, Optimization methodology assessment for the inlet velocity profile of a hydraulic turbine draft tube: part I - computer optimization techniques, J. Glob. Optim. 55 (1) (2013) 53-72. doi:10.1007/s10898-012-9946-8.

[29] F. Juretić, cfmesh: Advanced meshing tool (2017). URL cfMesh.com

[30] B. Marjavaara, T. Lundström, J. Wright, R. Kamakoti, S. Thakur, W. Shyy, Steady and unsteady CFD simulation of the turbine-99 draft tube using CFX and STREAM, in: Proceedings of the third IAHR/ERCOFTAC workshop on draft tube flow, no. 7, 2005, pp. 83-92. 
[31] U. Andersson, An experimental study of the flow in a sharp-heel draft tube, Ph.D. thesis, Luleå University of Technology, Sweden (2008).

[32] T. Vekve, An experimental investigation of draft tube flow, Ph.D. thesis, Norwegian University of Science and Technology, Norway (2004).

[33] M. Cervantes, T. Engström, Factorial design applied to CFD, J. Fluids Eng. 126 (5) (2004) 791-798. doi:10.1115/1.1792277.

[34] T. Ran, Z. Xuezhi, X. Buchao, W. Zhengwei, Numerical investigation of the flow regime and cavitation in the vanes of reversible pump-turbine during pump mode's starting up, Renew. Energy 141 (C) (2019) 9-19. doi:10.1016/j.renene.2019.03.

[35] B. Mulu, P. Jonsson, M. Cervantes, Experimental investigation of a kaplan draft tube - part I: Best efficiency point, Appl. Energy 93 (2012) 695-706. doi:10.1016/j. apenergy.2012.01.004.

[36] S. Daniels, A. Rahat, R. Everson, G. Tabor, J. Fieldsend, Shape optimisation of the sharp-heeled Kaplan draft tube: Part II - performance optimisation using a multi-objective Bayesian methodology, Renew. Energy (submitted).

[37] E. Catmull, R. Rom, A class of local interpolating splines, in: R. Barnhill, R. Riesenfeld (Eds.), Computer Aided Geometric Design, Academic Press, 1974, pp. 317-326. doi:10.1016/B978-0-12-079050-0. 50020-5.

[38] P. Jonsson, B. Mulu, M. Cervantes, Experimental investigation of a kaplan draft tube - part II: Off-design conditions, Appl. Energy 94 (2012) 71-83. doi:10.1016/j.apenergy.2012.01.032.

[39] N. Dahlbäck, Redesign of sharp heel draft tube - results from tests in model and prototype, in: E. Cabrera, V. Espert, F. Martínez (Eds.), Hydraulic Machinery and Cavitation: Proceedings of the XVIII IAHR Symposium on Hydraulic Machinery and Cavitation, Springer Netherlands, Dordrecht, 1996, pp. 985-993. doi:10.1007/ 978-94-010-9385-9_100. 
[40] C. Duarte, F. de Souza, V. dos Santos, Mitigating elbow erosion with a vortex chamber, Powder Technol. 288 (2016) 6-25. doi:10.1016/j. powtec.2015.10.032.

[41] Y. San, R. Thien, V. Chen, Numerical study on erosion of a pipe bend with a vortex chamber, Particulate Science and Technology 0 (0) (2018) 1-7. doi:10.1080/02726351.2017.1360973.

[42] H. Ghafori, M. Sharifi, Numerical and experimental study of an innovative design of elbow in the pipe line of a pneumatic conveying system, Powder Technol. 331 (2018) 171-178. doi:10.1016/j.powtec.2018. 03.022 